Eurostudia

\title{
Le paysage sonore
}

\section{Concevoir un patrimoine du son?}

\section{Karoline Schirmer}

Volume 8, numéro 1-2, 2012-2013

Paysages culturels de la modernité

Kulturlandschaften der Moderne

Cultural Landscapes of Modernity

URI : https://id.erudit.org/iderudit/1026635ar

DOI : https://doi.org/10.7202/1026635ar

Aller au sommaire du numéro

Éditeur(s)

Le Centre canadien d'études allemandes et européennes

ISSN

1718-8946 (numérique)

Découvrir la revue

Citer cet article

Schirmer, K. (2012). Le paysage sonore : concevoir un patrimoine du son?

Eurostudia, 8(1-2), 123-148. https://doi.org/10.7202/1026635ar

Tous droits réservés @ C Le Centre canadien d’études allemandes et européennes, 2014
Ce document est protégé par la loi sur le droit d'auteur. L'utilisation des services d'Érudit (y compris la reproduction) est assujettie à sa politique d'utilisation que vous pouvez consulter en ligne.

https://apropos.erudit.org/fr/usagers/politique-dutilisation/
Cet article est diffusé et préservé par Érudit.

Érudit est un consortium interuniversitaire sans but lucratif composé de l'Université de Montréal, l'Université Laval et l'Université du Québec à Montréal. Il a pour mission la promotion et la valorisation de la recherche. https://www.erudit.org/fr/ 
Karoline Schirmer

Universität Stuttgart

$\mathrm{L}$ e paysage sonore urbain est devenu un objet de recherche avec le compositeur et musicologue canadien R. Murray Schafer. Son résultat de recherche sur les effets néfastes du bruit sur les personnes a été intensément observé dans le monde de la musique. Mais les aspects sonores et acoustiques ne sont pas devenus des facteurs qui entrent en considération dans la conception de processus créateur. L'architecture moderne et l'urbanisme sont visuellement pensés et communiqués par les médias visuels : dessins, plans et modèles 3D. Ce que les musiciens, musicologues et acousticiens savent sur la psychologie perceptive et la psychoacoustique reste ignoré par la plupart des architectes et des urbanistes.

L'expérience sonore de l'espace et l'influence de l'acoustique spatiale sur le son et, inversement, l'influence du son sur l'expérience de l'acoustique spatiale pourraient être utilisées pour façonner le paysage sonore urbain. La plus grande partie de notre espace urbain n'est pas construit avec les aspects acoustiques à l'esprit, sauf certains espaces qui sont spécialement conçus pour l'écoute (salles de concert, théâtres etc.). De plus, la technologie moderne avec tous ses conforts est une source essentielle de l'ambiance sonore de la ville postindustrielle. Le bruit est devenu un problème de la modernité. Mais l'intensité du son n'est pas le seul aspect de l'environnement sonore urbain. Il est un domaine complexe qui se caractérise par trois éléments fondamentaux : la géométrie de l'espace, les matériaux et les objets sonores. Un de ces objets sonores est l'être humain lui-même. On se déplace dans cet espace, on perçoit et on crée des ondes sonores. La géométrie de l'espace change constamment en marchant à travers la ville. L'environnement urbain évolue en permanence avec les processus de développement et de transformation dans le cours du temps. L'homme doit s'adapter à cet environnement sonore changeant dans le temps et dans l'espace. Le son dense de la ville moderne avec ses bruits industriels a atteint une dimension jamais connu auparavant. La réaction humaine est la construction de barrières du son, la construction d'un monde 
acoustique privé avec l'aide de baladeur numérique ou d'une évasion dans les parcs.

Le but central de ce projet est la description d'une valeur patrimoniale de l'environnement sonore. Par contraste avec le modèle de Schafer je prendrai en considération que, en premier lieu, le paysage sonore urbain ne désigne pas seulement le son désagréable (bruit); et en second lieu, qu'il y a des outils et des moyens pour utiliser, manipuler et former le son urbain afin d'esthétiser l'environnement. Par conséquent, je voudrais traiter les questions suivantes de savoir comment est-ce que les villes modernes sont caractérisées par les aspects acoustiques, et comment est-ce que nous pouvons agir et façonner l'environnement sonore. Ensuite, j'évaluerai ce qui constitue la valeur propre d'un paysage sonore, et finalement, je tenterai de définir les critères selon lesquelles on pourra protéger quelques éléments significatifs d'un paysage sonore.

\section{L'ESPACE SONORE URBAIN : QUE DU BRUIT ?}

Tous ceux capables d'entendre connaissent l'effet émotif de la musique de film. La peur monte en nous quand nous entendons les trémolos des instruments à cordes graves. Même si l'image qui se présente sur l'écran n'a rien d'effrayant, ce sentiment naît en nous par l'arrière-plan musical. Mais il n'y a pas que la peur qui peut être produite par la musique de film. C'est en particulier dans les films d'amour ou dans les roadmovie, qu'à l'aide de la musique, on nous transmet tout un éventail d'ambiances et de sentiments positifs.

Le niveau sonore du film est donc capable d'ajouter à la profondeur visuelle une profondeur sémantique ou de changer de façon significative l'interprétation des images. Les musicologues sont bien conscients de cette capacité de la musique de générer certaines émotions. C'est en particulier pour cette raison que l'on a créé, dans le film, mais aussi dans les jeux informatiques ou dans les grands magasins, le niveau sonore.

Depuis environ 1955, il revient encore une autre fonction à la musique de film : à savoir, la fonction de rendre un film ou une série reconnaissable. Par son thème musical, il ou elle reste sans égal. Ce qui vaut pour le film pourrait de même être important pour la ville, mais manque encore de recherches et d'études suffisantes. Le paysage sonore d'une ville manque jusqu'ici d'un arrangement intentionnel, tout en restant largement créé par l'homme.

Le trafic des villes avec ses voitures et métros, les maisons qui reflètent les bruits, les rires des hommes, le bruit des enfants qui jouent sur un terrain de 
jeux, les bruits des marchés, la sonnerie des cloches - tout cela est le résultat d'actions humaines. Malgré tout, les architectes et constructeurs ne prennent pas vraiment conscience de ce fait. Quand il s'agit de construire et d'arranger une ville, il n'y a que peu d'aspects sonores qui sont pris en considération dans la planification. Ils se réduisent aux questions de barrières du son ${ }^{1}$.

Des méta-analyses montrent qu'en général, les recherches scientifiques sur l'environnement sonore ne prennent pour objet que les effets négatifs que produisent sur l'homme et l'animal les sons considérés comme bruit. Sur ce sujet Carles, López Barrio et de Lucio écrivent :

Environmental studies usually deal with sound in its noise or pollutant facet, and rarely as an informative or oriental element. The informative function of a landscape, however, is not circumscribed to this aggressive aspect. A landscape totally devoid of sound would certainly prove disturbing or unnerving ${ }^{2}$.

Cette réduction du soundscape (paysage sonore) à l'aspect du bruit et ainsi aux questions de l'abaissement de la qualité de vie est évidemment insuffisante, ainsi que l'aura, sans doute, illustrée la brève digression dans la signification de la musique de film. Le texte présent a pour but de montrer que le soundscape est bien plus que du bruit dans l'espace urbain. Il est conçu de façon à expliciter comment se constitue le paysage sonore d'une métropole moderne, mais aussi de régions champêtres, et comment il peut être décrit.

De plus, il faudra se demander dans quelle mesure le produit sonore de la vie de l'homme dans la métropole peut être considéré en même temps comme patrimoine culturel. De même que la musique de film peut avoir entre autres pour fonction d'être reconnue, le soundscape d'un paysage culturel peut être, lui aussi, doté de cette même fonction : le chant monotone des marchands à La Havane ou la sonnerie des cloches du Big Ben imposent à la ville en question une marque tout à fait unique.

\section{Le terme du soundscape}

Mais qu'est-ce qu'on entend par ce néologisme créé par contraction entre landscape et sound? Je fais référence ici à la définition proposée par Raymond Murray Schafer :

\footnotetext{
${ }^{1}$ Cf. Berliner Flughäfen, «Aktiver Lärmschutz », accédé le 6 août 2012, http://www.berlin-airport.de/DE/Umwelt/Fluglaerm/aktiverLaermschutz.html. ${ }^{2}$ J. L. Carles, I. López Barrio, J. V. de Lucio, "Sound influence on landscape values ", Landscape and Urban Planning 43,4 (1999), 191.
} 
Techniquement, toute partie de cet environnement est prise comme champ d'étude. Le terme s'applique aussi bien à des environnements réels qu'à des constructions abstraites, telles que compositions musicales ou montages sur bande, en particulier lorsqu'ils sont considérés comme faisant partie du cadre de vie ${ }^{3}$.

Sa description du concept du soundscape rend évident qu'il doit s'agir d'un terme ayant une portée très large. C'est la seule composition du mot qui suggère un rapport entre le son et l'environnement. Dans la publication fort recherchée d'Emily Thompson, Soundscapes of modernity, elle constate pour le soundscape comme objet de recherches, en analogie au terme du paysage :

I define the soundscape as an auditory or aural landscape. Like a landscape, a soundscape is simultaneously a physical environment and a way of perceiving that environment; it is both a world and a culture constructed to make sense of that world ${ }^{4}$

Alors qu'il faut entendre la perception du paysage surtout au sens de la diffusion et interprétation visuelles de stimuli d'un paysage physique, le soundscape est l'interprétation auditive d'un environnement physique et acoustique. Mais les deux termes contiennent un composant de sens évidemment spatial.

\section{Un néologisme : soundscape}

Le terme soundscape a été marqué dans le contexte de sciences sonores à l'orientation écologique, dans les années 1960. Son fondateur a été le compositeur et musicologue Raymond Murray Schafer 5 . Son World Soundscape Project, né au début des années 70, était consacré à la description systématique comparative de paysages sonores. L'intention du travail de Raymond Murray Schafer était la sensibilisation pour les conséquences négatives croissantes des nuisances sonores pour l'homme et l'environnement.

C'était un grand mérite et la position fondamentale de sciences sonores écologiques avait et a toujours sa justification. En effet, alors que nous pouvons nous protéger contre un trop d'impressions visuelles en détournant

\footnotetext{
${ }^{3}$ R.M. Schafer, Le paysage sonore (Paris : Lattés, 1979), 376.

${ }^{4}$ E. Thompson, The soundscape of modernity : Architectural Acoustics and the Culture of Listening in America, 1900-1933 (Cambridge, MA : MIT Press, 2004), 1.

${ }^{5}$ Cf. D. Morat, «Zwischen Lärmpest und Lustbarkeit. Die Klanglandschaft der Großstadt in umwelt- und kulturhistorischer Perspektive », dans Beiträge zum Göttinger Umwelthistorischen Kolloquium 2009-2010, dir. Bernd Herrmann (Göttingen :

Universitätsverlag Göttingen, 2010).
} 
le regard et en fermant les yeux, c'est bien plus difficile de se protéger contre les stimuli acoustiques. Cela a pour conséquence que nous sommes constamment contraints d'assimiler des informations acoustiques.

Raymond Murray Schafer, de même que d'autres scientifiques qui lui ont succédé, parmi lesquels Emily Thompson ${ }^{6}$ ou Daniel Morat ${ }^{7}$, renvoient le terme soundscape à un environnement sonore. Mais la véritable portée du terme soundscape ne naît que par son usage aussi bien pour des contextes réels que pour des espaces sonores abstraits, comme, par exemple, dans les compositions musicales, les montages sonores ou les enregistrements ${ }^{8}$.

Dans la définition d'Emily Thompson, les espaces sonores médialement transmis sont aussi explicitement définis. D'un côté, ces espaces sonores abstraits sont ceux qui, par la polyphonie composée, créent et transmettent un espace sonore. Bien particulière apparaît ici l'intégration d'espaces sonores techniquement reproduits. Il peut $s^{\prime} y$ agir aussi bien de photographies de coins de rue que de montages sonores qui font naître un rapport à l'espace. Il faut entendre l'élargissement conscient de la définition sur des soundscapes abstraits au sens d'une conséquence de la réalité de vie changée depuis ce que l'on appelle la modernité. Les progrès techniques du $19^{\text {ème }}$ et du $20^{\text {ème }}$ siècle ont changé fondamentalement les valeurs d'expériences essentielles dans l'espace et le temps.

Le téléphone permet de se parler, bien qu'il y ait des milliers de kilomètres qui séparent les interlocuteurs. La radio présente aussi une invention décisive de la modernité. Écouter un concert à la radio ou sur un disque signifie de sentir les mêmes espaces qu'un auditeur qui se trouve face à l'orchestre et écoute. Cette expérience de la relativité de l'espace et du temps est bien accueillie dans la définition d'un niveau descriptif qui, en tant qu'objet, n'a été considéré que depuis la moitié du $20^{\text {ème }}$ siècle.

Le présent travail va se concentrer surtout sur les soundscapes concrets et moins sur les espaces sonores tels qu'ils naissent au sein de compositions et de collages sonores. $\mathrm{Vu}$ que ce travail a pour but de déduire des chemins et mesures pour une amélioration qualitative des soundscapes, on décrit ici le soundscape non virtuel qui, en général, n'est pas sciemment arrangé.

\footnotetext{
${ }^{6}$ Thompson, The soundscape of modernity.

${ }^{7}$ Morat, « Zwischen Lärmpest und Lustbarkeit », 173-190.

${ }^{8}$ Voir la définition de Schafer, Le paysage sonore, 376.
} 


\section{Soundscape comme modèle descriptif de l'écologie sonore}

Le bruit n'est point une apparition de la modernité. Aecherli indique, par exemple, que l'on connaît déjà de l'Antiquité romaine des règles de construction pour les barrières de sons pour les chaudronniers ${ }^{9}$. Mais, entre temps, le niveau sonore dans les agglomérations a augmenté au point que, vers la moitié du $20^{\text {ème }}$ siècle, se sont formées des organisations internationales pour le combattre (en Allemagne, par exemple, le Deutsche Arbeitsring zur Lärmbekämpfung). Au Canada et aux États-Unis, le mouvement des écologues acoustiques est né autour de R. Murray Schafer, qui approche ce thème par la voie scientifique et expérimentale. Ce domaine de l'écologie acoustique est, selon Schafer "das Studium von Lauten in ihrer Beziehung zum Leben und zur Gesellschaft ${ }^{10}$.

Ce qu'a été le Bauhaus dans le domaine du design et de l'architecture, à savoir, la naissance du design industriel, Schafer le réclame désormais pour l'acoustique. Il considérait comme nécessaire l'arrangement conscient du soundscape, jugeant de façon négative le soundscape de la modernité. Selon lui, c'est la révolution industrielle à laquelle il faut imputer cette dégénérescence.

[Sie] brachte eine Vielzahl neuer Laute mit sich, und zwar mit unglücklichen Folgen für viele natürliche und menschliche Laute, die übertönt wurden. Diese Entwicklung wurde ausgeweitet, als die elektronische Revolution neue, eigene Wirkungen hinzufügte und Geräte für die Bündelung von Lauten einführte und dadurch diesen über Zeit und Raum hinweg eine verstärkte oder vervielfältigte Existenz gab ${ }^{11}$.

S'appuyant sur les explicitations de Walter Benjamin, les descriptions de Schafer sur la radio et la reproduction technique de la musique et de bruits produits par des hommes qui, sans connaître le silence, se laissent guider par des intérêts totalitaires, sont très négatives. En opposition à la sphère sonore Lofi de la modernité, où convergent beaucoup d'événements sonores et de bruits machinaux et techniques, il voit la sphère sonore Hi-fi marquée par les bruits de la nature et des signaux acoustiques singuliers. Alors que la sphère sonore Hi-fi se caractérise par sa qualité acoustique, la sphère sonore Lo-fi est de mauvaise qualité et se trouve surtout dans les espaces urbains de la modernité. Cette seule distinction entre Lo-fi et Hi-fi rend évident que le modèle descriptif de Schafer n'est pas suffisant pour le soundscape urbain. Il est vrai que le bruit est

\footnotetext{
${ }^{9}$ W. Aecherli, Umweltbelastung Lärm (Zürich/Chur : Rüegger, 2004), 10.

${ }^{10}$ R. M. Schafer, Klang und Krach (Frankfurt am Main : Athenäum, 1988), 249.

${ }^{11}$ Ibidem, 97.
} 
devenu un problème de la modernité, mais le paysage sonore de la ville ne se caractérise pas uniquement par des sons indésirables.

Ce n'est pas seulement à la musique concrète que la ville a offert un riche matériel sonore. La musique pop, telle que l'avant-garde d'aujourd'hui, se sert de sons caractéristiques de la ville dans les compositions musicales, vu qu'ils représentent une partie intégrante de notre réalité de vie et que, par conséquent, on les retrouve esthétiquement appliqués dans la musique.

Même si la prétention de Schafer d'un arrangement conscient du soundscape est légitime et toujours actuelle, à partir d'un modèle descriptif tellement négatif de notre environnement acoustique, on aurait du mal à obtenir un mémento pour améliorer la qualité acoustique. La critique face à la reproduction technique de sons ainsi que la dévalorisation du paysage sonore de notre époque et, par contre, la réévaluation de bruits de la nature ne proposent pas de points de départ pour une solution. La systématisation des éléments du soundscape, que Schafer propose dans sa publication The Tuning of the World de 197712, offre pourtant des catégories bien appropriées à la description de l'environnement acoustique. Schafer distingue entre trois catégories sonores essentielles: les sonorités maîtresses ou toniques; les signaux sonores; et les marqueurs sonores (soundmarks).

Le terme sonorité tonique dérive du domaine de la musique. Schafer y entend un caractère général de l'environnement acoustique. Les sonorités toniques se présentent comme combinaisons de sons que l'on n'entend pas nécessairement de façon consciente, mais qui marquent le caractère sonore général d'un endroit.

Grundtöne hört man nicht immer heraus; sie werden überhört, aber nicht übergangen, denn sie werden zu Hörgewohnheiten, die sich unbewusst festsetzen. [...] Die Grundtöne einer Landschaft werden von ihrer Geographie, ihrem Klima und ihrer Fauna bestimmt: von Wasser, Wind, Wäldern, Ebenen, Vögeln, Insekten und anderen Tieren ${ }^{13}$.

Comme sonorités toniques, on considère, par exemple, le bruit sourd du trafic d'une grande ville, le bourdonnement du frigidaire dans un appartement ou le chant des cigales en été en Crète.

La deuxième catégorie désigne des sons comme signaux. Il s'agit des sons que l'homme perçoit sciemment. En tant qu'exemple Schafer propose ici les cloches ou les sirènes. Dans le passage suivant, Schafer décrit un point

\footnotetext{
${ }^{12}$ R. M. Schafer, The Tuning of the World (Toronto : The Canadian Publisher, 1977).

${ }^{13}$ Schafer, Klang und Krach, 16.
} 
important: "Signallaute können in umfangreichen Codes organisiert werden, so daß sie die Übertragung komplizierter Botschaften an Empfänger, die sie dechiffrieren können, ermöglichen. " ${ }^{14}$ Ce que Schafer décrit comme "signal sonore » sera décrit de façon plus détaillée dans le chapitre L'interprétation de l'environnement acoustique de ce travail. Comme processus sémiotique de l'attribution de significations d'une communauté acoustique, on indique par les signaux sonores des concepts abstraits (voir figure 1). Un signal sonore renvoie par une référence à un composant matériellement existant de l'environnement. Comme signal sonore on peut considérer la sirène des pompiers ou la sonnerie du réveille-matin.

Comme troisième catégorie Schafer cite les marqueurs sonores. Cette catégorie sera également décrite dans le chapitre L'interprétation de l'environnement acoustique. Les marqueurs sonores sont liés à l'espace et peuvent devenir l'emblème d'un endroit ${ }^{15}$. La sonnerie du Big Ben et des cloches d'Hiroshima représentent des marqueurs sonores. La sonnerie des cloches représente tantôt un endroit concret qui sert de référence, tantôt un concept abstrait comme le commencement d'une messe voire même une communauté religieuse. Si l'on reprend les outils terminologiques de Schafer, la sonnerie des cloches présente d'un côté un signal, de l'autre aussi un marqueur sonore. À ce propos Schafer écrit :

Manche Orientierungslaute sind monolithisch und prägen ihre Signaturen der gesamten Gemeinschaft auf, wie zum Beispiel Kirchenoder Uhrenglocken, Hörner oder Pfeifen. Was wäre Salzburg ohne seinen Salvatore Mundi, Stockholm ohne sein Stadhuset- Glockenspiel, London ohne Big Ben? ${ }^{16}$

Les bruits alarmants du métro de Berlin au moment où se ferment les portes présentent, bien sûr, d'un côté, des marqueurs sonores, mais de l'autre, ils sont devenus entre-temps des soundmark pour le métro de Berlin, voire même pour la ville de Berlin.

Ce qui est intéressant, c'est que c'est précisément à cette catégorie de sons d'un soundscape que Schafer attribue une valeur culturelle particulière : "Wenn ein Orientierungslaut sich einmal herausgebildet hat, dann verdient er es, geschützt $z u$ werden, denn Orientierungslaute charakterisieren das akustische Leben einer

\footnotetext{
${ }^{14}$ Ibidem, 17.

${ }^{15}$ R. M. Schafer, « Soundscape - Design für Ästhetik und Umwelt » [1993], dans Der Aufstand des Ohrs - Die neue Lust am Hören, dir. Volker Bernius et al. (Göttingen :

Vandenhoeck \& Ruprecht, 2006), 147.

${ }^{16}$ Schafer, Klang und Krach, 292.
} 
Gemeinschaft ${ }^{17}$. Le paysage sonore urbain est touché par le problème du bruit, mais on trouve dans la ville de même des sons et sphères sonores qualitatifs auxquels on peut attribuer une valeur culturelle. Ce travail ne reprend donc pas le point de départ de Schafer de la mauvaise qualité du soundscape urbain. Son exigence d'un arrangement actif du soundscape reste pourtant toujours légitime aujourd'hui.

Pour cette raison, le présent travail essaie d'établir un mémento clair pour cet arrangement, à la base d'explications scientifiques sur l'importance des sons pour l'homme. Elles sont destinées à aider la planification urbaine et régionale à introduire des mesures qui permettent d'améliorer la qualité du soundscape. On demande une analyse exacte du soundscape sous l'aspect de la signification culturelle de ses composants, pour ne pas perdre des parties de notre patrimoine culturel. Malgré la dévalorisation surtout du soundscape urbain, la classification des différents niveaux sonores, proposée par Schafer, est fort utile. À l'aide de la distinction entre soundmarks, signals et key notes, il est possible de clairement désigner les processus de signes ainsi que la fonction de ces signes acoustiques dans une société.

\section{SOUNDSCAPE ET CULTURE}

Un nouveau modèle descriptif du soundscape : le modèle d'interaction

Ainsi que nous l'avons déjà vu dans les passages précédents, le soundscape est un terme spécifique de large portée. Les phénomènes décrits par ce terme et leur complexité sont tellement diversifiés qu'une systématisation paraît difficile. Dans la littérature, les phénomènes suivants sont associés au terme du soundscape :

- les phénomènes physiques ;

- les phénomènes de la psychologie perceptive ;

- l'environnement acoustique ;

- l'arrangement spatial de l'environnement acoustique (comme déterminant pour les caractéristiques acoustiques) ;

- les événements sonores dans un espace ;

- la production active des événements sonores par le corps humain ou d'autres objets ;

- la production acoustique de signes ;

- la création d'une communauté acoustique dans un espace ;

- la tradition musicale d'une communauté acoustique.

\footnotetext{
17 Ibidem, 17.
} 
En les examinant de plus près, on peut pourtant constater que tous ces phénomènes sont liés au fait que l'homme entre en interaction avec son environnement. La vraie difficulté, c'est que l'on peut entendre le soundscape aussi bien comme condition naturelle de cette interaction que comme produit de l'interaction entre l'homme et l'environnement.

En raison de la complexité des phénomènes décrits, le travail sur le soundscape a beaucoup de points communs avec d'autres sciences. Considérant le soundscape comme un modèle d'interaction, il s'y profile plusieurs niveaux. Le soundscape se présente, sur un premier niveau, comme un environnement acoustique qui consiste en événements (frissonnement) et en caractéristiques (texture du matériel et ses caractéristiques réflexives, etc.) physiques. On l'a déjà décrit dans les chapitres précédents.

Sur un deuxième niveau, un soundscape est une sélection et une interprétation de l'environnement acoustique. Cela ne veut pas faire référence au fait que l'environnement acoustique ne correspond pas à la représentation mentale, puisque l'homme perçoit, par exemple, différentes fréquences de façons différemment intensives. Le deuxième niveau du modèle décrit plutôt la sélection de stimuli acoustiques ; c'est-à-dire, la filtration d'une multitude de stimuli acoustiques en informations acoustiques plus et moins importantes.

Ce qui présente une information importante ou peu importante dépend de l'interprétation qu'en fait l'individu. Cela peut se faire par expérience ou dépendre de la situation actuelle. Cela peut également être déterminé par les buts que poursuit la personne. Restons-en au frissonnement dans les fourrés, dont nous avons déjà parlé : L'habitant d'une grande ville, à la recherche de tranquillité, peut en jouir, le ressentant comme un bruit d'arrière-plan qui détend; un promeneur errant à travers la nuit prendra peur, ne sachant comment interpréter les bruits ; un chasseur devient attentif, puisqu'il devine tout près quelque gibier; un couple amoureux n'en prend même pas conscience, étant tout à fait concentré à échanger des caresses.

D'un autre côté, on y entend, sur le deuxième niveau, des événements sonores auxquels une communauté acoustique attribue une certaine signification. Les premiers chants des oiseaux sont interprétés par une communauté acoustique comme le début du printemps, alors que l'on interprète le tonnerre comme la rage d'un dieu. Tous les passagers locaux connaissent la signification du signal de départ du métro de Berlin.

Le deuxième niveau décrit surtout la constitution des significations de l'environnement acoustique, laquelle contient une sélection du matériel 
acoustique ainsi qu'une codification/décodification. Une communauté acoustique sélectionne certains événements sonores de l'environnement acoustique et en conventionne la signification.

Sur le troisième niveau du soundscape comme modèle d'interprétation, on reconnaît un autre pas de l'interaction entre l'homme et l'environnement acoustique : la reproduction. On entend par là l'imitation de l'environnement acoustique. On y compte le sifflement de l'homme comme chant d'oiseau humain ou des mélodies qui reproduisent acoustiquement le bercement d'un bébé que l'on tient dans les bras. En même temps, l'imitation de l'environnement acoustique signifie la création de nouveaux matériaux acoustiques. On y obtient la rétroaction par rapport au premier niveau du modèle d'interaction. La création de nouveaux matériaux acoustiques modifie l'environnement acoustique physique.

\section{Soundscape en tant que cycle}

Le soundscape comme modèle d'interaction se conçoit comme un modèle cyclique. En outre, l'environnement acoustique ne change pas seulement à travers l'imitation par l'homme. Des processus naturels, pareils à ceux que l'homme initie, modifient l'environnement acoustique. La petite période glaciaire avait pour suite un changement de l'environnement acoustique. Quand, par exemple, en raison de changements climatiques, les hirondelles partent plus tard pour l'Europe centrale, cela change l'environnement acoustique. Si l'homme est capable de former des métaux, il peut produire des cloches. Quand le son de cloches métalliques pénètre l'environnement acoustique (premier niveau), l'homme peut profiter de ce matériau acoustique et donner au son des cloches une signification, telle que « début de la messe » (deuxième niveau). Mais il faut aussi activer les cloches et il peut y naître des suites particulières de la sonnerie des cloches qui, de leur côté, sont sélectionnées et interprétées d'une certaine façon, etc. Le soundscape est, par conséquent, un processus fort dynamique de l'interaction entre l'homme et son environnement acoustique.

\section{L'interprétation de l'environnement acoustique}

Un premier examen superficiel pourrait nous laisser croire que l'aspect extérieur visuel d'une ville domine l'idée que l'homme s'en était faite. Pour une majeure partie des hommes, cela pourrait être vrai. Mais pour la partie de la population qui souffre de problèmes auditifs, ce n'est certainement pas le cas. Mais même pour l'autre partie de la population générale, il n'existe pas d'études concernant cet aspect. Mais, même si les impressions optiques 
dominaient, cela ne signifie pas que les informations sonores perçues ne modèlent pas, elles aussi, l'image optique. Il suffit de penser à l'exemple de la musique de film pour se rendre vite compte qu'une impression visuelle est fort influencée par l'acoustique. Il existe un grand nombre d'études qui prouvent l'influence réciproque de données acoustiques et visuelles.

Parlitz et Colonius confirment l'importance de paramètres visuels pour le jugement et la perception de sons ${ }^{18}$. Ils supposent que, lors du processus mental d'assimilation, les stimuli auditifs et visuels coïncident dans les neurones multi-sensoriels. Il $s^{\prime} y$ fait la comparaison des deux stimuli qui guident la fonction de la perception spatiale et de l'orientation. D'autres études scientifiques prouvent également une forte assimilation entre les stimuli visuels et auditifs lorsqu'il s'agit de localiser un endroit. L'image des rues de New York qui, d'en haut d'un gratte-ciel, semblent de larges gorges, s'accompagne d'un certain frémissement du bruit de la circulation, qui coïncide avec la profondeur de l'espace, visuellement perçue, et contribue à l'image complète d'une grande ville agitée avec des monuments qui se dressent vers le ciel. Carles, López Barrio et de Lucio ont examiné le jugement de certaines combinaisons de stimuli visuels et auditifs, et ont constaté que les stimuli qui ne vont pas trop ensemble étaient ressentis plutôt comme désagréables. Dans ce cas-ci, le son était ressenti comme du «bruit » ${ }^{19}$.

Ainsi que nous l'avons déjà explicité dans le chapitre Le premier composant: Sound, l'environnement acoustique fournit de nombreuses informations importantes sur la texture de l'environnement. L'homme compare ces informations acoustiques avec celles que lui fournissent les autres sens. Selon ce que l'on a déjà décrit, elles soutiennent leur propre localisation dans l'environnement, c'est-à-dire : Où suis-je ? Où se trouvent d'autres objets ou sujets dans cet « espace acoustique »? Quelle est la texture de ces objets ?

Dans le secours que l'on porte aux concitoyens aveugles, on utilise déjà sciemment des informations acoustiques. Il existe ainsi dans beaucoup de grandes villes en Allemagne des feux de circulation qui, dotés d'un cliquetis, indiquent à l'aide de signaux acoustiques quand on peut traverser une rue sans danger. Devant les écoles pour les enfants amblyopes, il y a des voies d'acheminement acoustiques qui indiquent aux élèves l'entrée de la cour de l'école, ainsi que d'autres sons à l'aide desquels ils peuvent parcourir, sans

\footnotetext{
${ }^{18}$ D. Parlitz et H. Colonius, «Effect of visual stimulation on auditory detection », dans Contribution to Psychological Acoustics, dir. August Schick (Oldenburg : BIS-Verlag, 1993), 387-395.

${ }^{19}$ Carles, López Barrio, et de Lucio, « Sound influence on landscape values », 191 et suiv.
} 
danger, le chemin jusqu'à l'entrée de l'école. La possibilité d'utiliser ces informations est un point essentiel quand on réfléchit sur les paysages sonores urbains. Un son perçu présente toujours un lien entre l'individu et le milieu dans lequel il se trouve. Pour entendre le son que l'on peut percevoir dans un certain endroit, il faut que nous y soyons (sauf pour les reproductions techniques). Ainsi, l'impression sonore que nous laisse un endroit peut avoir également une fonction de reconnaissance.

Nous avons déjà décrit dans le chapitre L'espace sonore urbain: que $d u$ bruit? que la musique de film peut avoir cette fonction de reconnaissance. Mais on peut également reconnaître un endroit en raison ses expériences acoustiques. En anglais, on appelle cela «acoustemology ${ }^{20}$. À l'aide du souvenir acoustique, lié à un endroit, un espace devient un endroit reconnaissable, ou bien le point fixe d'un espace. La spécificité acoustique d'un endroit permet à l'homme de le percevoir en tant qu'entité et de le distinguer d'autres. La constitution de points fixes est un mécanisme mental général. À ce propos Ehrich écrit :

Aus der Infinitheit des Raums folgt für die räumliche Orientierung, daß wir uns Fixpunkte setzen, die es erlauben, die Unendlichkeit in einer der begrenzten Wahrnehmung zugänglichen Art zu strukturieren ${ }^{21}$.

Les sons ou les combinaisons de sons peuvent donc porter une signification pour avoir une certaine forme acoustique qui les distingue des autres, et qui ne se présente que dans un ou plusieurs endroits précis. Ainsi, le son constitue pour l'individu un espace ou certaines caractéristiques d'un espace. Cela peut être, par exemple, le craquement spécifique des lames du plancher dans une maison, lequel permet à un auditeur de reconnaître celle-ci. Au moment où plusieurs individus se partagent le savoir sur cette référence du stimulus acoustique, ils forment une communauté acoustique ${ }^{22}$. Cette communauté acoustique peut être, dans un petit cadre, la famille qui habite une maison et partage le savoir sur le craquement spécifique des lames, comme particularité de sa maison. Une communauté acoustique peut aussi constituer une plus grande collectivité qui interprète le craquement spécifique des lames comme référence à un certain type de maisons.

\footnotetext{
${ }^{20}$ K. Kato, "Soundscape, cultural landscape and connectivity », Sites, New Series 6,2 (2009), 84.

${ }^{21}$ V. Ehrich, « Die temporale Festlegung lokaler Referenz », dans Raumkonzepte in Verstehenskonzepte: Interdisziplinäre Beiträge zu Sprache und Raum, dir. Christopher Habel et al. (Tübingen : Niemeyer, 1989), 2.

${ }^{22}$ Cf. Kato, «Soundscape, cultural landscape and connectivity », 84 .
} 
Les sons peuvent représenter des aspects naturels, culturels et historiques d'un endroit, et se trouver en rapport avec le savoir, les aptitudes ou la spiritualitée ${ }^{3}$. Dès qu'un son ou une combinaison de sons sont devenus pour un groupe une référence convenue, il y naît une communauté acoustique, liée à cet endroit. Schafer appelle ces sons « soundmarks » :

Klänge, die schon lange an einem bestimmten Ort sind, nenne ich soundmarks. Wie Wahrzeichen (landmarks) definieren sie seine wesentliche Eigenart und machen ihn damit einmalig ${ }^{24}$.

Des exemples de soundmarks seraient le bruit de portes en fer de maisons traditionnelles qui se ferment, la sonnette du tramway caractéristique de St.Etienne et certaines chansons de l'équipe nationale dans le stade local.

Les soundmarks ne doivent pas nécessairement représenter l'endroit en tant qu'un tel, mais peuvent également, ainsi que nous l'avons déjà mentionné au début, se référer aux aspects de cet endroit. Kato décrit un troisième niveau des sons comme éléments sémantiques :

Thirdly, sound can be a carrier of ethics and spirituality. Sound can carry traditional and historical knowledge of living in place and communicate the complexity of knowledge, ethics and connectivity to those outside of their world. Sounds also carry social memories about a place $^{25}$.

Cela signifie que les sons peuvent renvoyer au savoir et aux concepts abstraits. Ce savoir peut concerner l'endroit en tant qu'un tel, ou par contre, être un savoir qui ne se partage qu'à un certain endroit. Le craquement des lames renvoie, par conséquent, à un endroit concret, à savoir, la maison ou plusieurs maisons d'un même type.

Si les sons d'un environnement acoustique peuvent renvoyer à des concepts abstraits, ils peuvent également assumer la fonction d'endroits de souvenirs abstraits. Selon Pierre Nora, les lieux de mémoire peuvent être en particulier des points de cristallisation, autres que matériels ${ }^{26}$, d'une mémoire

\footnotetext{
${ }^{23}$ Ibidem.

${ }^{24}$ Schafer, « Soundscape - Design für Ästhetik und Umwelt », 147.

${ }^{25}$ Kato, "Soundscape, cultural landscape and connectivity », 84 .

${ }^{26}$ Par autre que matériel ne doit pas être nié à cet endroit-ci que les phénomènes acoustiques n'auraient pas de dimension physique et matérielle, mais, par rapport aux endroits de souvenirs acoustiques, ce terme décrit la cristallisation non-visuelle et mobile.
} 
ou identité collective ${ }^{27}$. De même, le son peut faire partie des usages, dans le contexte de certains lieux de mémoire. Les fascistes espagnols fêtent l'anniversaire de Franco sur sa tombe qui est un monument commémoratif gigantesque, et y chantent des chansons fascistes de l'époque de Franco.

\section{Les conditions et les changements du soundscape}

Les sensations auditives peuvent aussi être sciemment manipulées. Ainsi, un grand espace peut sembler, par sa forme et ses matériaux, beaucoup plus petit, parce que le son s'absorbe de façon extraordinairement rapide. De même, on peut conférer à un petit espace une acoustique pareille à ce que l'on a dans les églises, en utilisant des matériaux unis, durs et des murs droits. Ce dont on tient compte pour arranger des espaces enfermés, pourrait être également considéré pour l'arrangement de soundscapes. Quel effet doit produire une ville sur son visiteur? Quelles émotions doit-elle déclencher? Comment arranger une valeur de reconnaissance acoustique?

Le soundscape d'une ville ou d'un autre espace est déterminé par trois éléments différents : la géométrie de l'espace; les matériaux et, les sources sonores. Par géométrie de l'espace on entend la forme d'un espace. Une rue qui d'en haut d'un gratte-ciel semble une gorge étroite et profonde provoque d'autres réflexions sonores qu'une route large et plate. Les espaces ronds produisent une autre acoustique que les espaces angulaires.

La géométrie de l'espace et l'arrangement de la surface de la ville sont créés presque uniquement par les hommes. Il est vrai que, pour son arrangement, l'homme s'adapte aux données naturelles, en intégrant, comme par exemple à Phœnix, Arizona, une colline dans l'image de la ville, en profitant de vallées comme lieu d'implantation, tel qu'à Stuttgart, ou bien en faisant des fleuves les artères d'une ville comme à Berlin. Mais les rues et les maisons ont été projetées et construites par la main humaine. Et même les rares sources de bruits naturels et d'éléments spatiaux «naturels", tels des arbres et des oiseaux qui chantent, apparaissent en majeure partie sous le contrôle de l'homme.

Ainsi, dès la moitié du 19ème siècle, on a déjà aménagé, dans de nombreuses métropoles du monde, des parcs au milieu de la ville ${ }^{28}$. L'exemple

\footnotetext{
${ }^{27}$ Cf. Pim Boer et al., Europäische Erinnerungsorte 3 : Europa und die Welt (München :

Oldenburg Verlag, 2012), 265.

${ }^{28}$ J. Jacsman et R. Shilter, Landschaftsplanung : Aufgaben, Grundsätze, Konzepte und Methoden für eine ökologisch orientierte Raumplanung (Zürich : vdh Hochschulverlag, 1995), 206.
} 
le plus célèbre est, sans doute, Central Park de New York qui a été aménagé en 1854: un petit espace de nature et lieu de repos dans une ville agitée et bruyante. En même temps, Central Park était un projet élaboré par des architectes paysagistes, selon le modèle d'un paysage idéalisé de l'Amérique du nord ou de l'Angleterre ${ }^{29}$.

Central Park ne présente pourtant pas de mesures de protection contre le bruit, destinées à refouler les bruits de la ville, mais doit permettre de vivre la nature. La conception romantique de la force salutaire de la nature, telle que

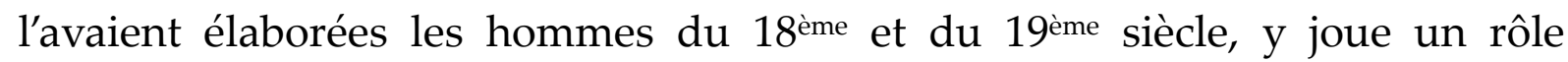
central. Central Park a été aménagé en premier lieu comme élément naturel visuel et devait illustrer la beauté du paysage des Etats-Unis. En même temps, le parc implentait, dans le paysage urbain, un soundscape contrastif qui, par sa texture, son caractère acoustique, assume la fonction de lieu de repos. Les bruits naturels, comme le bruit de l'eau, le chant des oiseaux et le frissonnement du feuillage, sont devenus des éléments certes bien organisés, mais caractéristiques du soundscape de New York. Ainsi, Central Park est, certes, visuellement conçu, mais un point essentiel du parc, pour les habitants de la ville, réside dans son acoustique.

Au tournant du $19^{\text {ème }}$ et du $20^{\text {ème }}$ siècle, il y eut une réorientation dans la planification urbaine, vers une intégration projetée et aménagée de la nature dans l'espace urbain. Ainsi que le montrent, par exemple, les cités de la modernité de Berlin, déclarées patrimoine culturel par l'UNESCO, au début de 20 ème siècle, on peut reconnaître une tendance qui s'éloigne de jardins privés et se dirige vers les espaces verts publics ${ }^{30}$. Cela ne vise pas seulement une réévaluation esthétique et structuration de l'espace. La construction sociale comprenait également le repos dans la nature. L'espace vert ou le parc offrent, en particulier par la texture/le caractère de leur soundscape, un endroit de repos pour les habitants de la ville.

Aujourd'hui, il s'y ajoute encore d'autres réflexions qui invitent les planificateurs à intégrer des espaces verts dans le concept de «ville». On a bien compris que, ce que l'on appelle la vitrification du terrain, c'est-à-dire le fait de boucher la surface du terrain par des monuments, des rues et des chemins, a une influence sur l'écosystème, le climat local ainsi que les plantes

\footnotetext{
${ }^{29}$ S. Low, D. Taplin et S. Scheld, Rethinking Urban Parks : Public Space and Cultural Diversity (Austin, TX : University of Texas Press, 2005), 20.

${ }^{30}$ Cf. Deutsche UNESCO-Kommission, «Siedlungen der Berliner Moderne », accédé le 14 juillet 2012, http://www.unesco.de/berliner-siedlungen.html.
} 
et les animaux ${ }^{31}$. Mais ce n'est pas seulement l'aménagement de l'espace qui a une influence directe sur la texture, le caractère du soundscape. Comme il fut déjà évoqué, les matériaux de construction, sur lesquels se modifient les ondes sonores, influencent également les caractéristiques des réflexions. Une ville produit d'autres sons quand elle est construite en bois, que quand elle est construite en pierre naturelle. C'est aussi le revêtement de la chaussée que l'on utilise dans une ville qui est déterminé par l'homme. Le pavé cause plus de bruit quand une voiture y passe que l'asphalte.

La troisième catégorie qui constitue un soundscape se présente par les sources sonores. C'est en particulier en ville que les événements sonores sont directement ou indirectement provoqués par l'homme : les voitures, les motos, la langue, le rire, les pas, la musique. Le paysage sonore d'une ville est soumis à des changements permanents. De nouveaux monuments sont construits, d'autres sont démolis; on change et ajoute les matériaux, on modifie les formes, on construit des allées ou intègre des stationnements. Une multitude de plans et de projets réalisés dans la grande ville entraînent un changement du paysage sonore, même si, en général, on n'y avait pas réfléchi. Et pourtant, le changement de la géométrie de l'espace se fait encore de façon relativement lente par rapport au changement des événements sonores.

L'évolution dans le domaine de la technologie, l'introduction et l'évolution de la circulation urbaine publique, la densité de la ville, constamment croissante, le transfert des quartiers résidentiels dans les zones moins centrales de la ville, et bien d'autres encore, modifient les événements sonores dans l'espace urbain. Peter Payer décrit avec l'exemple de Vienne comment les événements sonores ont changé depuis la fin du $19^{\text {ème }}$ siècle ${ }^{32}$. Il voit en particulier dans les renouvellements techniques et dans la motorisation croissante des causes importantes du changement du paysage sonore urbain.

Von all den ineinander verwobenen und verschobenen Klängen gab es einen Geräuschkomplex, der die Großstadt wie kein anderer repräsentierte: das Rollen und Rattern, Quietschen und Klingeln der Straßenbahn, die sich zum städtischen Verkehrsmittel par excellence entwickelte ${ }^{33}$.

\footnotetext{
${ }^{31}$ L. Haag, «Wie hoch sind die Versiegelungsgrade in Berlin wirklich? - Ein Methodenvergleich » (Diplomarbeit, Institut für Landschaftsarchitektur und Umweltplanung, Technische Universität Berlin, 2006).

32 P. Payer, « Der Klang von Wien. Zur akustischen Neuordnung des öffentlichen Raumes », Österreichische Zeitschrift für Geschichtswissenschaft 4 (2004), 105-131.

${ }^{33}$ Payer, « Der Klang von Wien », 129.
} 
Ainsi, au début du 20 ème siècle, le tramway électrique est devenu à Vienne et dans beaucoup d'autres villes européennes le symbole de la grande ville. Il symbolisait la ville, parce qu'il signifiait une innovation technique essentielle et un enrichissement pour la mobilité en ville. Pendant plusieurs siècles, il constituait le pendant des fiacres qui continuaient à marquer le paysage urbain. Par contraste avec ces charrettes tirés par les chevaux, le tramway électrique était marquant et son bruit machinal était particulier, ayant un son "moderne", son moteur constituant la véritable innovation: c'était une machine. Aujourd'hui, on n'entend plus le tramway comme symbole de la grande ville. Il a été remplacé par d'autres moyens de transport, surtout la voiture qui, depuis, domine les rues de la ville. Désormais, c'est le grondement collectif des voitures dans les rues des métropoles gigantesques, qui ressemblent du quarantième étage d'un gratte-ciel à de larges gorges, qui est devenu le symbole de la grande ville.

\section{Soundscape comme produit de l'interaction avec l'environnement}

Emily Thompson a élargi le terme du soundscape, en indiquant sa culturalité : "A soundscape, like a landscape, ultimately has more to do with civilization than with nature, and as such, it is constantly under construction and always undergoing change. ${ }^{34}$ Si l'on entend par culture l'ensemble des actes humains qui peuvent être réalisés de différentes manières, il faut entendre le soundscape comme culturellement déterminé. Jusqu'ici, nous avons dégagé, pour le terme du soundscape surtout, deux composants essentiels: les événements sonores et l'espace.

La définition de Catherine Guastavino nous fournit un autre aspect qui a été examiné, à l'aide de méthodes psycholinguistiques, le jugement de soundscapes familiers dans les grandes villes françaises ${ }^{35}$. Elle écrit: «The notion of soundscape, first introduced by Schafer is commonly defined as an environment of sound with emphasis on the way the environment is perceived ${ }^{36}$. Ainsi, elle décrit le soundscape par deux dimensions. Dans sa première dimension, il comprend l'environnement acoustique qui se compose de phénomènes physiques et non-virtuels. Dans sa deuxième dimension, la perception et l'interprétation des mêmes phénomènes. On reconnaît cette même distinction entre les deux dimensions dans le modèle d'interaction, élaboré dans ce travail ; les deux dimensions y correspondent aux niveaux 1 et

\footnotetext{
34 Thompson, The Soundscape of Modernity, 2.

${ }^{35}$ C. Guastavino, « The Ideal Urban Soundscape : Investigating the Sound Quality of French Cities », Acta Acustica united with Acustica 92,6 (2006), 945-951.

${ }^{36}$ Ibidem, 945.
} 
2 que nous avons décrits. Dans le modèle que j'ai développé, il s'y ajoute, par contre, une autre dimension : la (re)production. Ce n'est pas seulement que l'homme se trouve dans un environnement acoustique, qu'il le perçoit et l'interprète, mais en plus, organise activement le soundscape par l'aménagement de l'espace (rues, maisons, jardins, etc..), et la production de bruits et de sons (pas, voitures, langue, chant, etc.). Les deux dernières dimensions sont culturellement déterminées, aussi bien en ce qui concerne la production du soundscape que son interprétation. Un paysage, aussi un paysage sonore, suppose l'interprétation par l'homme et est, par conséquent, lié aux valeurs esthétiques.

Mais, la sélection et l'interprétation de l'environnement acoustique dépend bien sûr de la texture, du caractère de l'environnement. Le crépitement de la pluie a une autre signification pour un habitant du désert que pour l'habitant d'une ville portuaire où il pleut souvent. L'arrivée de martinets avec leur chant spécifique ne peut être interprétée comme début du printemps qu'à condition que ces oiseaux fassent partie de ce soundscape. En examinant le troisième niveau du soundscape, la (re)production, on y voit tout de suite l'aspect de culturalité. Les traditions musicales constituent une forme de la reproduction et une (re-)formulation artistique de l'environnement acoustique. La tonalité des traditions musicales de l'Europe centrale est fort différente de celles que l'on trouve en Inde ou en Afrique. C'est de même l'aménagement spatial acoustique sous forme de traditions architectoniques qui diffère selon les cultures. Les événements sonores que l'homme produit en forme directe ou indirecte sont culturellement différents. La possibilité de distinguer entre les soundscapes est donc une forme de la diversification culturelle de l'humanité.

\section{Soundscape comme patrimoine culturel}

L'exemple du tramway qui, à la fin du $19^{\text {ème }}$ siècle et au début du $20^{\text {ème }}$ siècle, a marqué le soundscape de la grande ville, devenant son symbole, nous dirige vers deux questions centrales : jusqu'à quel point faut-il entendre soundscape comme patrimoine culturel ? Dans quelle mesure devrions-sous le protéger, le réévaluer et le conserver?

Il n'est pas toujours facile de répondre à ces questions et la réponse dépend toujours du point de vue de celui qui la cherche. Il faut réfléchir sur la question de savoir en raison de quelle valeur culturelle une chose doit être érigée en patrimoine culturel. Cela peut, mais ne doit pas être sa dimension acoustique. Le tramway de cette époque-là n'a pas seulement une carrosserie qui paraît historique, mais c'est aussi le bruit du tramway en mouvement qui 
permet de le localiser dans le temps. Si l'on considère donc le tramway comme patrimoine culturel en raison de son rôle particulier comme symbole de la grande ville moderne, le son fait absolument partie du patrimoine culturel du tramway. Si, par contre, on considère le tramway comme patrimoine culturel en raison de ses innovations sur le niveau du design, son son serait sans pertinence. On peut en conclure que le son ne doit pas être nécessairement un critère pour déterminer le patrimoine culturel. Si, pourtant, un son contient d'importantes informations de reconnaissance, à l'aide desquelles on peut localiser un objet culturellement précieux dans son contexte, il fait également partie du patrimoine culturel.

En recourant aux possibilités techniques des nouveaux médias, beaucoup de musées ont trouvé entre-temps des voies d'exposer des pièces d'exposition originales. Par des médias virtuels, la pièce d'exposition peut être complémentée, voire même complètement remplacée. D'un côté, cela favorise les pièces d'exposition qui peuvent être mieux conservées et soignées pour les générations suivantes. De l'autre, il est plus facile de transmettre la fonction et le rôle du patrimoine culturel dans le contexte original. Elle peut compenser la décontextualisation des pièces d'exposition du musée. Un tramway qui, hors fonction, ne transporte plus les personnes de A vers $\mathrm{B}$, parce qu'il se trouve au musée, devient une conserve de musée qui manque d'authenticité. Le contexte d'origine n'existe plus et, pourtant, il doit s'en garder une petite partie.

Mais, dans le cas du tramway, ce dilemme bien connu du musée se laisse diminuer, entre autres, par un baladeur numérique. Un enregistrement original ou imité du soundscape d'un tramway roulant permet de faire revivre et de rendre sensible le contexte historique.

Schafer est d'avis que ce sont en particulier les marqueurs sonores ${ }^{37}$ qui portent une valeur culturelle et méritent d'être protégés ${ }^{38}$. Il énumère toute une suite de marqueurs sonores qui, peu à peu, disparaissent du soundscape :

- «das Scharren der schweren Metallstühle auf den Fußböden der Pariser Kaffeehäuser;

- das helle Zuschlagen der Türen alter Wagons der Pariser Métro, dem ein scharfes Klicken folgt, wenn der Riegel in die Schließposition einklingt [...]

- die hellen Glöckchen der Pferdetaxis in Konja [...] ${ }^{39}$.

\footnotetext{
${ }^{37}$ Pour le terme du marqueur sonore, voir le chapitre Soundscape comme modèle descriptif de l'eécologie sonore, supra.

${ }^{38}$ Schafer, Klang und Krach, 292.

${ }^{39}$ Ibidem, 293.
} 
L'exemple déjà évoqué de la sonnerie et du grincement des rails du tramway de Berlin a aussi été un marqueur sonore, que le soundscape de Berlin a déjà perdu.

Mais, les signaux sonores fort conventionnels peuvent aussi être d'une valeur culturelle particulière. Ils se laissent moins attribuer à un endroit fixe, mais font partie de la culture d'une communauté. Les klaxons continus d'une voiture de mariage présentent, par exemple, une tradition acoustique. C'est en particulier la communauté d'origine turque de Berlin qui célèbre cette tradition sur les rues principales de Belin, en honneur des nouveaux mariés. Cette tradition acoustique est donc d'une grande valeur pour cette communauté et est devenue une partie du soundscape de Berlin.

\section{Soundscape comme paysage culturel}

Ce que nous avons explicité jusqu'ici suggère l'hypothèse selon laquelle le soundscape se laisse décrire comme paysage culturel. Il faut pourtant être bien attentif en employant le terme du paysage culturel, vu que, dans les différentes sciences, il existe différentes conceptions de ce qu'il faut comprendre. Dans son emploi par l'UNESCO et selon la façon de voir de la géographie et de l'écologie du paysage, il est réduit à un rapport réciproque entre les paysages naturels réels et l'homme «au sens de paysages utilitaires ${ }^{40}$. La définition terminologique de l'UNESCO y apparaît comme terme juridique. Particulièrement dans les sciences culturelles et humaines, le terme s'emploie de façon beaucoup plus ouverte, utilisé pour les paysages urbains, jusqu'aux espaces virtuels liés à une dimension culturelle ${ }^{41}$. Nous avons déjà expliqué que non seulement la forme de la production sonore diffère dans les différentes cultures, mais aussi que l'interprétation de sons et de bruits dépend de la culture. Mais il s'y ajoute encore un autre aspect. Le jugement esthétique de sons et de bruits diffère aussi selon la culture (ainsi que la situation et l'individu).

La ville de Naples à la population dense est habitée par des hommes qui aiment bien communiquer à travers de grandes distances. Les cris, les grondements et le rire bruyant des Napolitains amusent, dans un premier moment, un Allemand et lui semblent exotiques. Si, par contre, Naples devenait son (nouveau) milieu résidentiel, il ressentirait l'intensité des voix humaines probablement comme du bruit. On peut douter si les Napolitains lui

\footnotetext{
${ }^{40}$ T. Reeh, Kulturlandschaft verstehen (Göttingen : Göttinger Universitätsverlag, 2012), 13 et suiv.

${ }^{41}$ Ibidem.
} 
donnent raison: on supposera plutôt que ce tapis de voix napolitain est devenu une caractéristique ainsi qu'un signe de reconnaissance de la ville. En tant que Napolitain, on s'identifie avec cet entourage sonore.

Les communautés acoustiques qui partagent certains genres d'interprétation de sons et disposent de certaines traditions de la production sonore se partagent en environnement acoustique. $\mathrm{Vu}$ que les expériences acoustiques doivent d'abord être faites pour partager des conventions acoustiques, on peut, en général, attribuer aux communautés acoustiques un certain espace. On peut désigner cet espace du soundscape d'une communauté acoustique par le terme du paysage culturel. Que l'on reprenne l'exemple du grincement des lames qu'une communauté acoustique entend au sens de référence à un certain type de maisons (bâtiment ancien). La dimension spatiale de cette communauté acoustique est l'espace dans lequel existe ce type de maisons et de plus la convention de l'association (" grincement des lames » $=$ «bâtiment ancien »).

Ainsi que nous l'avons montré dans le chapitre sur L'interprétation de l'environnement acoustique, l'environnement acoustique de la communauté acoustique, ou bien le soundscape d'une communauté acoustique, constitue un système de signes culturellement déterminé. Pour cette raison, on peut entendre un certain soundscape ou ses parties comme patrimoine culturel intangible (intangible cultural heritage) ou patrimoine de ses composants.

L'Accord sur la protection du patrimoine culturel intangible de l'UNESCO de 2003 donne pour le terme $d u$ patrimoine culturel intangible l'explication suivante :

On entend par "patrimoine culturel immatériel" les pratiques, représentations, expressions, connaissances et savoir-faire - ainsi que les instruments, objets, artefacts et espaces culturels qui leur sont associés - que les communautés, les groupes et, le cas échéant, les individus reconnaissent comme faisant partie de leur patrimoine culturel. Ce patrimoine culturel immatériel, transmis de génération en génération, est recréé en permanence par les communautés et groupes en fonction de leur milieu, de leur interaction avec la nature et de leur histoire, et leur procure un sentiment d'identité et de continuité, contribuant ainsi à promouvoir le respect de la diversité culturelle et la créativité humaine. Aux fins de la présente Convention, seul sera pris en considération le patrimoine culturel immatériel conforme aux instruments internationaux existants relatifs aux droits de l'homme, 
ainsi qu'à l'exigence du respect mutuel entre communautés, groupes et individus, et d'un développement durable ${ }^{42}$.

Dans la convention, on peut lire à la suite que la langue peut être un «vecteur du patrimoine culturel immatériel ${ }^{43}$. Que la langue soit un système de signes qui diffère de ceux d'autres communautés linguistiques, ce n'est pas nouveau. Mais, ainsi qu'on l'a montré dans le paragraphe précédent, les formes acoustiques peuvent aussi renvoyer à des concepts sémantiques. D'un côté, nous produisons les éléments acoustiques qui portent un sens et aménageons l'environnement acoustique, de l'autre, une communauté acoustique dispose de concepts spécifiques de l'interprétation de son environnement acoustique.

On peut entendre selon deux façons le soundscape comme patrimoine culturel intangible: Premièrement, le soundscape est une espèce d'association d'une communauté acoustique. Cette communauté dispose de codes et de concepts de décodification. L'attribution de sens est une systématisation de l'environnement acoustique qui diffère de celles d'autres communautés acoustiques. Deuxièmement, le soundscape est l'environnement acoustique d'une communauté acoustique, dans laquelle certains signes acoustiques sont des éléments qui portent le sens pour les concepts culturels.

Le flamenco est le composant d'un soundscape. La communauté acoustique vit surtout en Andalousie, en Espagne, où elle partage et pratique sa tradition de la production sonore. La communauté acoustique dispose du savoir sur les sons et la signification du flamenco. En même temps, le flamenco est beaucoup plus qu'une forme d'expression acoustique, car ce qu'il exprime et signifie exactement fait également partie du patrimoine culturel intangible. Il renvoie, en même temps, à d'autres concepts culturels, comme par exemple, la cohabitation des sexes et le rôle des gitanos dans la société ibérique, etc. En 2012, l'UNESCO a déclaré le flamenco comme patrimoine culturel intangible ${ }^{44}$.

Kato ${ }^{45}$ indique en plus que le soundscape d'une communauté acoustique peut être considéré comme interaction entre l'homme et l'environnement. On y aménage, d'un côté, un environnement acoustique donné par la nature, par des formes d'expression acoustiques, et de l'autre, une communauté

\footnotetext{
42 UNESCO, "Convention pour la sauvegarde du patrimoine culturel immatériel 2003 », accédé le 7 août 2012, http://unesdoc.unesco.org/images/0013/001325/132540e.pdf.

${ }^{43}$ Ibidem

${ }^{44}$ UNESCO-Intangible Cultural Heritage, «Flamenco », accédé le 14 août 2012, http://www.unesco.org/culture/ich/en/RL/00363.

${ }^{45} \mathrm{Kato}$, « Soundscape, cultural landscape and connectivity ».
} 
acoustique en fait un système de signes qui, en outre, peut porter le savoir culturel, les aptitudes ou les usages.

Mais, ce n'est pas seulement par une communauté acoustique locale que le soundscape devient un paysage culturel. C'est en particulier l'aspect de l'interaction entre l'homme et son environnement naturel qui est d'une importance centrale pour le terme du paysage culturel selon l'UNESCO. Depuis le décret de l'Orientation devant guider la mise en auvre de la Convention du patrimoine mondial de 1992, le terme du paysage culturel est devenu un terme juridique. Selon l'article 1 de l'accord, les paysages culturels représentent les « ouvrages combinés de la nature et de l'homme ${ }^{46}$.

Depuis 2008, on a encore stipulé les genres de paysages culturels qui peuvent être reconnus par l'UNESCO, soient les paysages aménagés (jardins et parcs); les paysages en développement (comme paysages vivants ou de vestige); et les paysages associatifs que l'on relie avec des événement et des pratiques culturels ${ }^{47}$. Suivant cette catégorisation de l'UNESCO, le soundscape en tant que produit de l'interaction entre l'homme et la nature, ou bien l'environnement (voir chapitre 3 Soundscape et culture) est un paysage culturel. Il est vrai que les événements acoustiques, le savoir sur ceux-ci et les concepts de signification ne sont pas des traces visibles dans un paysage. Mais la définition de l'UNESCO comprend aussi bien des paysages culturels associatifs que les interactions culturelles non visualisées dans un paysage naturel.

Mais, la définition de l'UNESCO est surtout un instrument juridique pour conserver le patrimoine culturel. Ce travail n'a pas pour but de déclarer un certain soundscape comme patrimoine culturel mondial. Il s'agit plutôt de montrer que le soundscape est un produit culturel qui a une valeur et qui résulte de l'interaction entre l'homme et son environnement.

Jusqu'ici, le soundscape n’a été traité dans la littérature spécialisée que comme concept négatif, alors il est grand temps de révéler les parallèles avec le concept de patrimoine culturel. La réflexion sur le soundscape comme patrimoine culturel doit stimuler une nouvelle pensée jusqu'à un point de vue

\footnotetext{
46 UNESCO, « Paysages Culturels », accédé le 14 août 2012, whc.unesco.org/fr/PaysagesCulturels.

${ }^{47}$ UNESCO-ICOMOS Documentation Centre, « World Heritage Cultural Landscape », February 2009, accédé le 14 août 2012, http://www.icomos.org/landscapes/Declarados\%20PM\%20y\%20bibliograf\%EDa.pdf; et UNESCO, « Paysages Culturels ».
} 
différencié, c'est-à-dire non plus uniquement négatif, mais basé en même temps sur des aspects positifs.

\section{CONCLUSION}

On a montré que le soundscape peut être décrit comme un modèle d'interaction cyclique entre l'homme et son environnement (naturel), où l'homme et les sons s'influencent réciproquement. Ce processus devient cyclique par la réaction de l'homme face à son environnement acoustique ; lui, qui change et influence le soundscape à son tour, créant ainsi un nouvel environnement qu'il jugera adéquat ou non, ouvrant sur de nouvelles interventions. L'environnement sonore du paysage culturel de la modernité comme le quelle dans la ville moderne est le résultat de l'adaptation de l'homme à son environnement.

Dans les travaux de Raymond Murray Schafer, desquels ont émergé de nombreuses perspectives sur le concept de soundscape, les paysages sonores ont été considérés, jusqu'ici, surtout sous l'aspect d'intervention contre le bruit, au sens d'une écologie sonore. Or, la réflexion sur le soundscape comme patrimoine culturel peut stimuler une nouvelle conception de l'intervention jusqu'à un point de vue différencié, c'est-à-dire non plus uniquement négatif, mais basé en même temps sur des aspects positifs.

L'aménagement de l'environnement sonore ne contient pas seulement des aspects de contrôle par la réduction du volume sonore, étant donné que le bruit ne s'est pas révélé être une catégorie objective. Le point de départ de ce travail est la première idée selon laquelle on peut considérer l'environnement sonore non pas uniquement comme négatif, mais aussi comme positif. Les bruits de machines ne doivent pas être par définition désagréables, et à l'inverse, pris pour acquis que la musique classique ou un concert de musique rock seront ressentis comme agréables.

Un son ressenti comme désagréable ou comme du bruit est le résultat d'un jugement esthétique, donc un processus d'évaluation en valeur. Il peut en résulter du coup, différents points de départ pour un aménagement du soundscape, non seulement sous l'aspect d'une diminution du niveau sonore. La recherche de solutions au problème des nuisances sonores est légitime, mais ne suffit pas à elle seule. Le présent travail offre une nouvelle perspective sur le soundscape : la valorisation, et si nécessaire, la patrimonialisation du son de l'environnement représentent une condition fondamentale pour intervenir sur la qualité sonore de la ville moderne. 
148 Paysages Culturels de la Modernité

La ville, ceci inclut la grande ville moderne, recèle toute une multitude de beaux sons agréables : la sonnerie du tramway, le brouhaha du café, le jeu de ballon des enfants dans la cour de la caserne de Berlin, etc. Pour cela, il est important d'envisager collectivement des outils qui permettront la reconnaissance du paysage sonore comme patrimoine. 